\title{
Study of deformation and failure in an anisotropic rock with a three-dimensional discrete element model
}

\author{
Yulong Zhang ${ }^{\mathrm{a}, \mathrm{b}}$, Jianfu Shao ${ }^{\mathrm{a}, \mathrm{b}, *}$, Géry de Saxcéb $^{\mathrm{b}}$, Chong Shi ${ }^{\mathrm{a}}$, Zaobao Liu ${ }^{\mathrm{c}}$ \\ ${ }^{a}$ Key Laboratory of Ministry of Education for Geomechanics and Embankment Engineering, Hohai University, \\ Nanjing, 210098 China \\ ${ }^{b}$ Laboratory of Multi-physics and Multi-scale Mechanics, FRE2016, CNRS, University of Lille, 59650 \\ Villeneuve d'Ascq, France \\ ${ }^{c}$ Key Laborato of Ministry of Education on Safe Mining of Deep Metal Mines, College of Resources and Civil \\ Engineering,Northeastern University, Shenyang, 110819, China
}

\begin{abstract}
This paper is devoted to numerical analysis of deformation and failure of transversely isotropic rocks. A three-dimensional discrete element (DEM) model is proposed in the framework of the particle flow code (PFC). The anisotropic rocks are characterized by a matrix phase in which non-persistent weak layers are embedded. The mechanical behavior of the matrix is described by a non-linear bond contact model while that of weak layers by a smooth joint contact model. The local elastic and strength properties in the bond and smooth joint models are calibrated from the corresponding macroscopic properties. Numerical predictions are compared with experimental data obtained from triaxial compression tests performed on a clayey rock, Tournemire shale. The anisotropic behavior of both elastic modulus and failure strength is well reproduced. The influence of confining stress on the failure strength of shale rock is also captured. A series of three dimensional numerical simulations are further performed for different orientations of weak layers and confining stresses. The effect of weak layers and confining stress on both local cracking process and macroscopic failure mode of rock samples is investigated and discussed.
\end{abstract}

Keywords: Anisotropic rocks, Three dimensional discrete element model, Weak layers, Smooth joint contacts, Nonlinear bond model, Confining stress effect

\section{Introduction}

Inherent anisotropy is one of the most representative features of many rocks, especially in sedimentary and metamorphic rocks. The micro-structure of anisotropic rocks is generally characterized by oriented fabric elements such as bedding or weak layers, oriented mineral grains 5 and pores, as well as oriented joints. The deformation and strength properties of anisotropic

\footnotetext{
*Corresponding author: jian-fu.shao@polytech-lille.fr
} 
rocks are strongly dependent on the loading orientation with respect to these fabric elements.

A large number of experimental studies have been conducted on different kinds of anisotropic rocks. It is not easy to make an exhaustive review of all previous studies and only some representative ones are mentioned here. For instance, Niandou et al. [1] have investigated the numerical methods, the particle flow code $(\mathrm{PFC})$ is one of the widely used ones. The basic principles of this method can be found in $[17,18]$. With this method, it is possible to describe the individual motion of grains and the local deformation of contact surfaces. Although ini- 

of anisotropic rock samples.

In the present paper, the following sign convention will be adopted. The compressive normal stress is denoted as a positive value and the tensile normal stress as a negative one. 


\section{Generation of anisotropic rock samples}

In the framework of discrete element method for rock-like materials, the continuum rock mass is replaced by an assembly of discrete particles which are connected by bonded contact interfaces. In the case of isotropic materials, the contact interfaces are randomly distributed in space without preferential orientations. In anisotropic materials, mechanical properties are orientation dependent. We assume that the anisotropic properties are due to the presence of oriented weak layers. The orientation of weak layers is defined by the angle $\theta$ with respect to $x-y$ plane in the global coordinates frame, as illustrated in Fig. 1 . In order to define the loading orientation with respect to weak layers, a local coordinates frame is also introduced. At the microscopic level, anisotropic rocks are represented by an isotropic rock mass in which weak layers are embedded. The isotropic rock mass is composed of a random assembly of particles with bonded contacts. The weak layers are represented by smooth joint contacts. Therefore, the following numerical procedure is proposed for the generation of anisotropic rock samples. An isotropic distribution of bonded contacts is first constructed. A number of smooth joint contacts are then inserted in the selected orientation $\theta$, which is defined by the angle with respect to the $x-y$ plane (see Fig. 2). For the insertion of smooth joint contacts in the isotropic rock matrix, a number of reference contacts are first selected by the following method. The orientation of each contact is defined by the vector line linking the centers of two neighboring particles $O_{1}-O_{2}$ as shown in Fig. 3. The orientation of the linking line is given by the angle $90-\theta$ with respect to the $x-y$ plane. The angle $90-\theta$ is further decomposed into two angles, respectively as $\alpha$ in the $y-z$ plane and $\beta$ in the $x-z$ plane. If two angles $\alpha$ and $\beta$ both meet the specified values, then the contact between these two neighboring particles is chosen as the reference contact $O$. Each reference contact is further taken as the center point of a smooth joint zone which is assumed to be penny shaped (see Fig. 3). The size of each smooth joint zone or weak layer zone is further defined by its radius $R$. Finally, all contacts inside the penny shaped weak layer zone are considered as the smooth joint contacts, such as $C_{1}$ and $C_{2}$ in Fig. 3. 


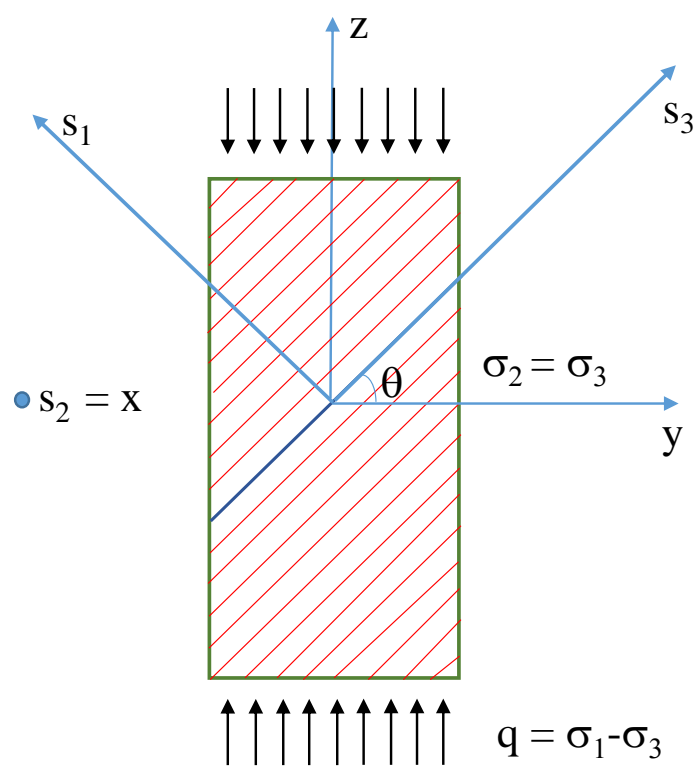

Fig. 1: Definition of weak layer orientation

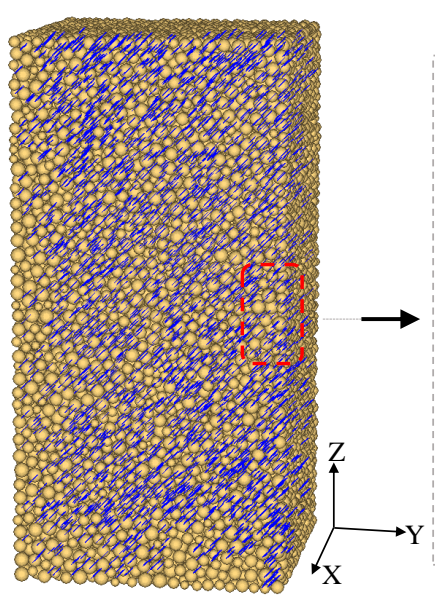

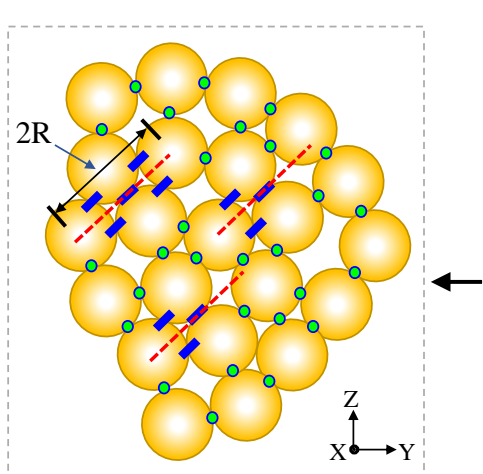

(b) Anisotropic material

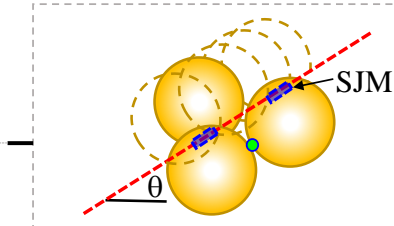

(c) Weakness layer

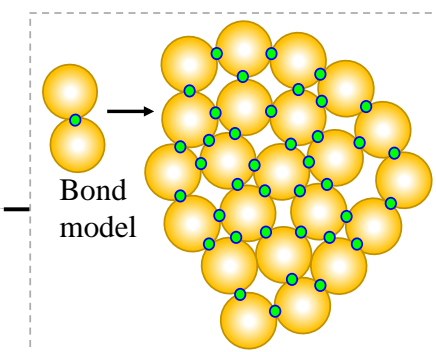

(d) Isotropic matrix

Fig. 2: Illustration of anisotropic rock sample generation 


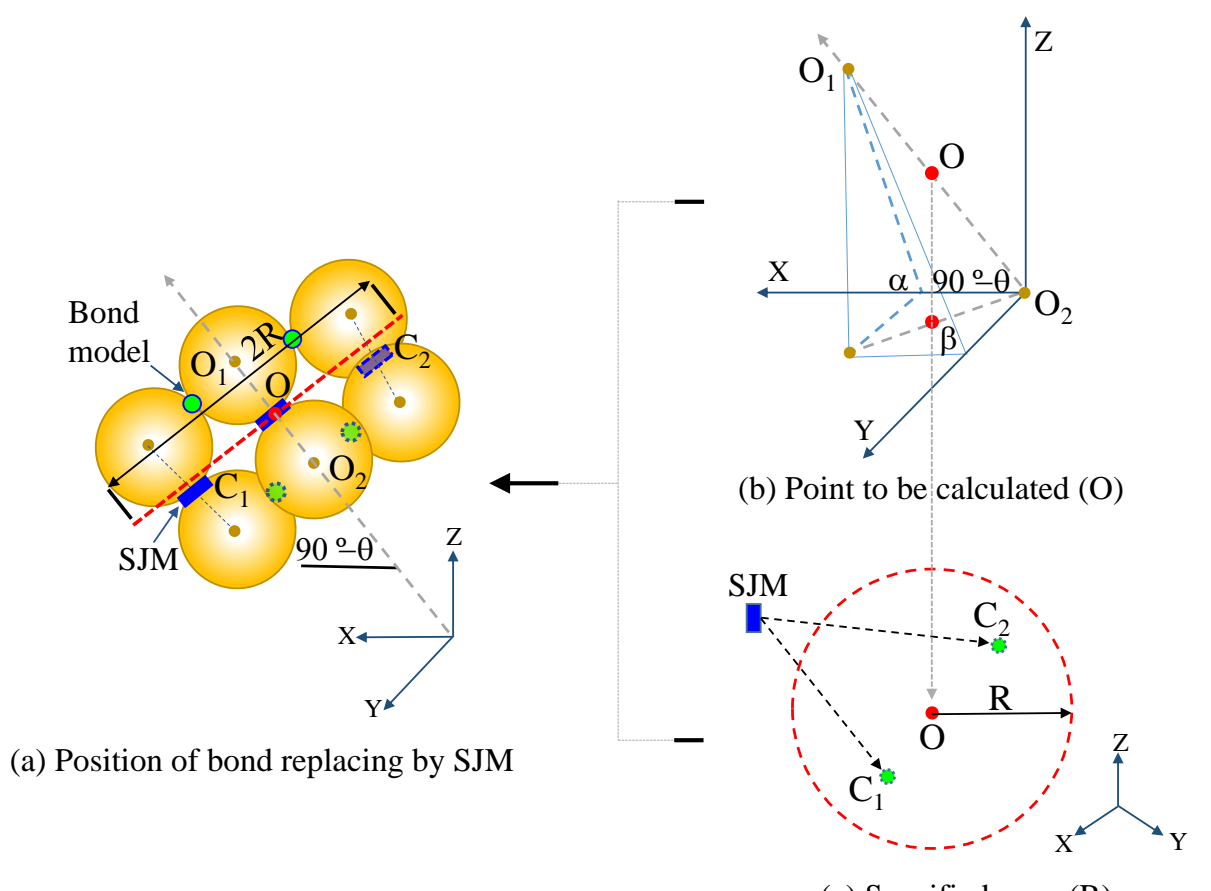

(c) Specified zone (R)

Fig. 3: Illustration of choice of smooth joint contacts

The spatial distribution of contact number is dependent on the shape and size of numerical sample. If a constant radius $R$ is used for all weak layers, the number of smooth joint contacts inside each weak layer zone will depend on its orientation. In order to avoid this inconvenient geometrical effect and obtain a quasi constant number of smooth joint contacts when the shape and size of rock samples are modified, we assume that the radius of weak layer zone $R$ is a function of its orientation angle $\theta$ as follows:

$$
\left\{\begin{array}{l}
R=\left(1+f_{R}(\theta)\right) R_{0} \\
f_{R}(\theta)=r_{R}|\cos (\pi / 2-\theta)|
\end{array}\right.
$$

$R_{0}$ denotes the radius of the weak layer zone with $\theta=0 . r_{R}$ is a dimensionless parameter introduced here to obtain a quasi constant number of smooth joint contacts for different orientations of weak layer zones. Its value depends on the shape and size of numerical sample. For a three dimensional cubic sample, as the size of sample is identical along three axes, the radius of weak layer zone should also be the same in all orientations. Therefore, in this particular case, one gets $r_{R}=0$.

By using the numerical procedure described above, seven anisotropic rock samples are generated. The size of all the samples is $40^{*} 40^{*} 80 \mathrm{~mm}^{3}$. The average radius of particles is taken as $1 \mathrm{~mm}$. The choice of this value is motivated by the fact that the average radius of particles should be small enough with respect to the size of samples, for instance 40 to 50 times smaller. Further, according to the size of rock samples considered here, the values of $R_{0}$ and $r_{R}$ are 
respectively taken as $1 \mathrm{~mm}$ and 0.3 . Seven different orientations of weak layers are considered, ranging from $0^{\circ}$ to $90^{\circ}$ with a constant interval of $15^{\circ}$. Three representative samples are shown in Fig. 4. In these Figures, we also show the spatial distribution of bond contacts and smooth joint contacts. One can see that the number of smooth joint contacts in all anisotropic rock samples is nearly the same and about 400. This indicates that the proposed generation procedure is able to correctly reproduce anisotropic rock samples containing weak layers in different orientations.

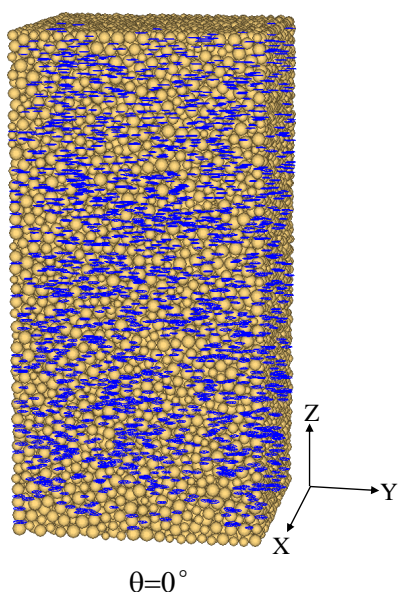

$\theta=0^{\circ}$

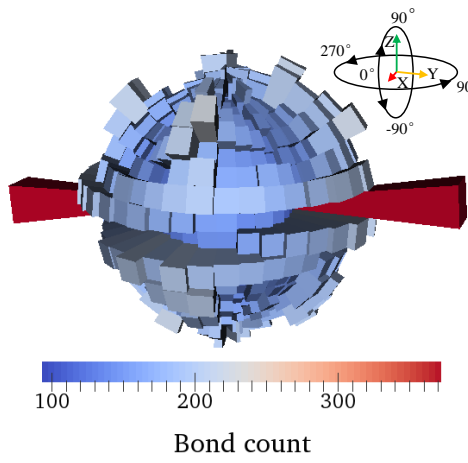

$\theta=0^{\circ}$

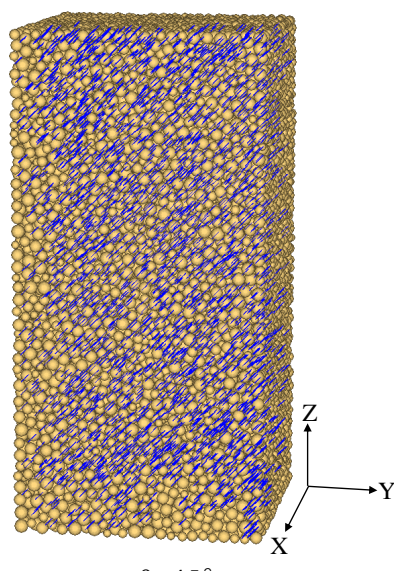

$\theta=45^{\circ}$

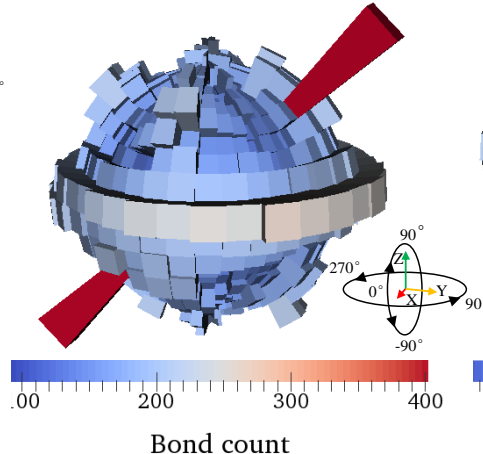

$\theta=45^{\circ}$

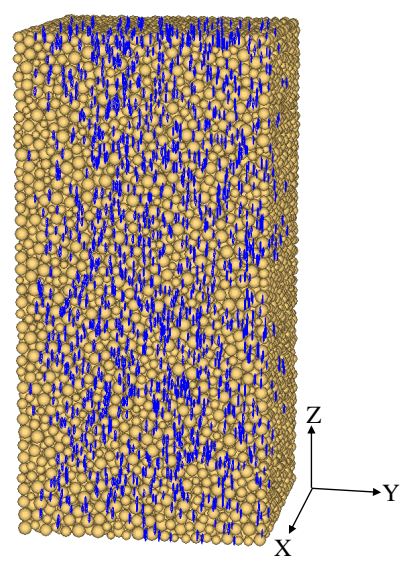

$\theta=90^{\circ}$

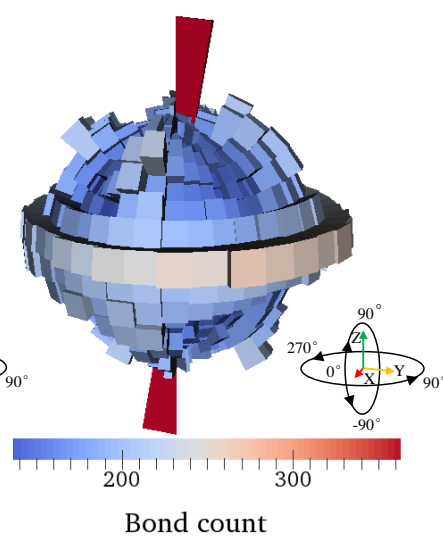

$\theta=90^{\circ}$

Fig. 4: Three representative anisotropic rock samples and corresponding spatial distribution of bond contacts and smooth joint contacts

\section{Presentation of contact models}

In order to describe the mechanical behavior of anisotropic rocks, we propose here a unified mechanical model for the bond contact in the rock matrix and the smooth joint contact in the weak layers. The proposed model is then implemented in the standard 3D Particles Flow Code. The formulation of the model is presented in this section. 


\subsection{Bond contact model}

Before failure of bond contacts, their mechanical behavior is described by an elastic model. In the present study, the emphasis is put on the failure process of bond contacts. The elastic behavior of bond contacts is described by a simple linear model already implemented in the PFC3D code [37]. The local force-displacement relations are given by:

$$
\begin{gathered}
F_{n}=k_{n} u_{n} \\
\Delta F_{s}=-k_{s} \Delta u_{s}
\end{gathered}
$$

The proposed nonlinear failure criterion is illustrated in Fig. 5. Two failure mechanisms are considered, respectively the tensile failure and shear failure. The tensile failure occurs when the normal contact force $F_{t, f}$ exceeds the normal strength $\varphi_{t}$. For the shear failure, it is known that the shear strength of contact is dependent on the compressive normal force $F_{n}$. As a consequence at the macroscopic scale, the macroscopic shear strength of rocks is strongly sensitive to confining stress. In order to consider this feature, the shear strength is described by a bi-linear function of the normal contact force. When the normal contact force is less than the transition threshold $\varphi_{c r}$, the shear strength is defined by the cohesion $\varphi_{s}$ and the frictional angle $\phi_{1}$. When the normal contact force is higher than $\varphi_{c r}$, a second frictional angle $\phi_{2}$ is introduced to define the shear strength under high confining stress. In general, we have $\phi_{2}<\phi_{1}$ due to the fact that initial micro-cracks in rocks are closed and the asperity of some micro-cracks can be destroyed when the confining pressure is high. Physically, the transition normal contact force $\varphi_{c r}$ defines two regimes of shear failure in rocks respectively under low and high confining stress. The value of $\varphi_{c r}$ can be identified from the macroscopic failure surface 
obtained from triaxial compression tests under a large range of confining stress, as mentioned in [36]. The failure criterion for contact interface is then expressed in the following form:

$$
\begin{gathered}
F_{t, f}=\varphi_{t}, \text { tensile failure } \\
F_{s, f}= \begin{cases}0 & , \quad F_{n}<\varphi_{t} \\
\varphi_{s}+F_{n} \tan \phi_{1} & , \quad \varphi_{t} \leq F_{n} \leq \varphi_{c r} \\
\varphi_{s}+\varphi_{c r}\left(\tan \phi_{1}-\tan \phi_{2}\right)+F_{n} \tan \phi_{2} & , \quad F_{n} \geq \varphi_{c r}\end{cases}
\end{gathered}
$$

As a difference with classical soil-like materials, most rocks are cohesive materials. In the particle-based models as that used here, discrete particles are bonded by contact interfaces. When a contact interface is broken, its tensile strength or inherent cohesion is assume to be completely destroyed. The breakage of contact interface can be seen as an equivalent process to micro-cracking in rocks. It is known that the surfaces of micro-cracks in most rocks are not fully smooth. Under a compressive normal stress, those micro-cracks are closed and exhibit some residual shear strength. This residual shear strength is here interpreted by a residual value of the friction coefficient of contact interface denoted by $\phi_{r}$. The residual strength envelop is shown in Fig. 5 and is described by the following criterion:

$$
F_{s, r}= \begin{cases}0 & , F_{n} \leq 0 \\ F_{n} \tan \phi_{r} & , F_{n}>0\end{cases}
$$

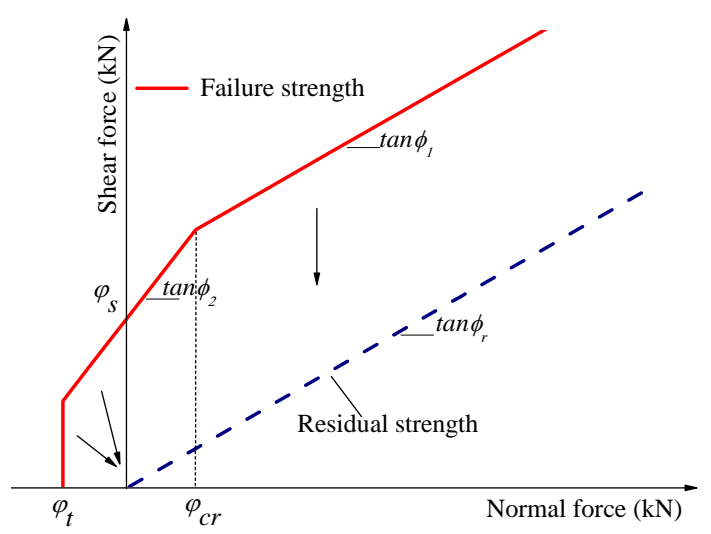

Fig. 5: Peak and residual strength envelopes of bi-linear criterion for bond contact and smooth joint contact

\subsection{Smooth joint contact model}

In the unified model proposed in this study, the failure of smooth joint contacts is described by the same relations as those for the bond contacts, given in 4, 5 and 6 . However, the strength 
parameters (cohesion and frictional coefficients) of the smooth joint contacts are deteriorated with respect to those of the bond contacts. Furthermore, after failure, the relative motion of particles around a broken smooth contact is different with that of particles around a broken bond contact. A comparison between two kinds of contacts is given in Fig. 6. For a broken bond contact, two neighboring particles are restricted to rolling one to another around the contact interface as shown in Fig. 6(a). However, for a broken smooth joint contact shown in Fig. 6(b), the pair of particles can slide along the orientation of weak layer $\theta$ and move each to other with a limited overlap.

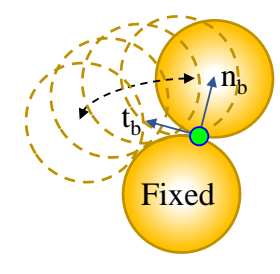

(a) Bond model

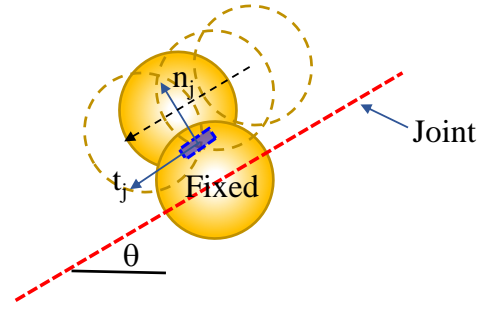

(b) Smooth joint model

Fig. 6: Relative motions of two neighboring particles around a broken bond contact and a broken smooth joint contact

\subsection{Anisotropy of elastic stiffness}

In particle-based models, the elastic behavior is described by the local normal and shear elastic stiffness coefficients of contact surfaces, respectively denoted as $k_{n}$ and $k_{s}$. The issue here is to calibrate these local elastic coefficients from the macroscopic elastic properties measured from laboratory tests. For this purpose, a number of numerical investigations have been performed, for instance $[39,40]$. It is found that it is possible to establish some empirical relations between the local elastic coefficients and macroscopic elastic modulus and Poisson's ratio. The local tangent elastic stiffness $k_{s}$ can be related to the normal one $k_{n}$ by the ratio coefficient $k_{r}$ such that $k_{s}=k_{r} \cdot k_{n}$. Further, the ratio $k_{r}$ depends on the macroscopic Poisson's ratio and varies in the range 1.0-3.0. These previous results are here adopted. However, in isotropic materials, the local elastic stiffness coefficients are kept constant for all contacts of different orientations. This is not the case for anisotropic materials. The elastic stiffness coefficients $k_{n}$ and $k_{s}$ of a contact should vary with its relative orientation with respect to the weak layers. For instance, the elastic stiffness of contacts parallel to the weak layers is generally smaller than 
that of contacts in other orientations. Inspired by [41], the following relations are proposed:

$$
\left\{\begin{array}{l}
k_{n}=\left(1+r_{k} \cdot f(\phi, \theta)\right) \cdot k_{n}^{0}, \quad k_{s}=\left(1+r_{k} \cdot f(\phi, \theta)\right) \cdot k_{s}^{0} \\
k_{n}^{0}=2 E_{c} \cdot\left(R_{1}+R_{2}\right), k_{s}^{0}=k_{r} \cdot k_{n}^{0} \\
f(\phi, \theta)=a /\left(b+(1-b) e^{-c \delta}\right)-a, \delta=\arctan |\tan (\phi-\theta)|
\end{array}\right.
$$

$\phi$ is the orientation angle of contacts. $k_{n}^{0}$ and $k_{s}^{0}$ denote the elastic stiffness of contacts for $\phi=\theta$. As mentioned above, the value of $k_{n}^{0}$ is calculated from the elastic modulus $E_{c}$ and the radius of two neighbouring particles, denoted by $R_{1}$ and $R_{2}$. The value of $k_{s}^{0}$ is related to that of $k_{n}^{0}$ through the ratio coefficient $k_{r}$.

Three parameters $a, b$, and $c$ are introduced in the function $f(\phi, \theta)$ to account for the spatial variation of local elastic stiffness of contacts. This function defines the ratio between the stiffness of contacts with the direction angle $\phi$ and that with the angle $\theta$. The variation of $f(\phi, \theta)$ with the relative angle $\delta$ is presented in Fig. 7 for different values of $b$ while the values of $a$ and $c$ are equal to 0.1 . The maximum value of $f(\phi, \theta)$ defines the anisotropy degree of elastic stiffness and it is sensitive to the value of $b$. The maximum value of $f(\phi, \theta)$ is found for $\delta=90^{\circ}$. It means that the maximum of elastic stiffness is found for the contacts which are perpendicular to weak layers. At the macroscopic scale, with the help of the function $f(\phi, \theta)$, it is possible to describe the variation of elastic modulus with loading orientation in anisotropic rocks. The values of three parameters $a, b$ and $c$ can be identified from experimental values of elastic modulus measured in uniaxial or triaxial compression tests performed in different loading orientations.

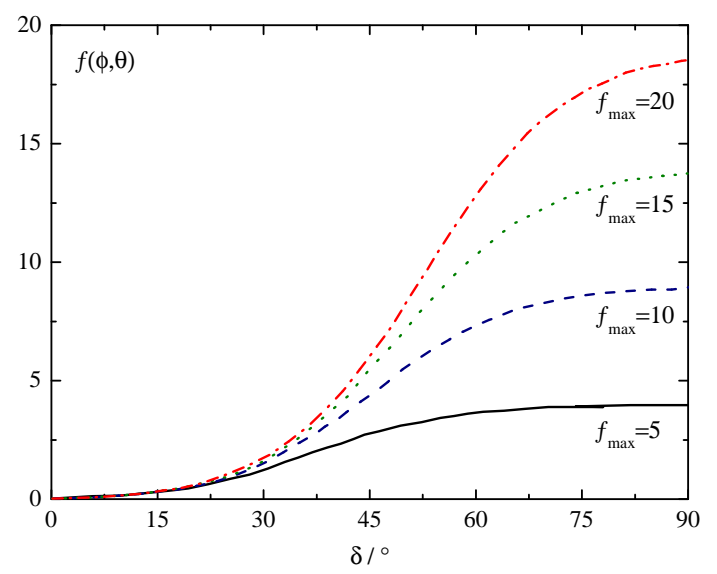

Fig. 7: Illustration of the function $f(\phi, \theta), \delta=\arctan |\tan (\phi-\theta)|$

\subsection{Anisotropy of mechanical strength}

Similarly to the elastic stiffness, the mechanical strength parameters of a contact are also dependent on its orientation with respect to the weak layers. According to previous studies 
[36, 42], among five parameters involved in the failure criterion of contacts defined above, the tensile strength $\varphi_{t}$ and the pure shear strength $\varphi_{s}$ have a more significant effect than other parameters on the macroscopic mechanical strength. Therefore, it is assumed that their values vary with the relative orientation angle of contacts with respect to the weak layers orientation $\phi-\theta$. A similar variation function as that for the elastic stiffness is used, that is:

$$
\left\{\begin{array}{l}
\varphi_{t}=\left(1+r_{s} \cdot f(\phi, \theta)\right) \cdot \varphi_{t 0}, \varphi_{s}=\left(1+r_{s} \cdot f(\phi, \theta)\right) \cdot \varphi_{s 0} \\
f(\phi, \theta)=a /\left(b+(1-b) e^{-c \delta}\right)-a, \delta=\arctan |\tan (\phi-\theta)|
\end{array}\right.
$$

165 exactly perpendicular to the weak layers. Therefore, it is necessary to introduce a small angle tolerance for the selection of smooth joint contacts. In the present study, we have taken $\pm 5^{\circ}$ 
and $\pm 4^{\circ}$ as the tolerances of the angles $\alpha$ and $\beta$ respectively. There is no theoretical criteria $\tan \phi_{1}$ and $\tan \phi_{2}$ as well as the transition threshold $\varphi_{c r}$ are fitted from experimental peak 
stresses. Note that in some cases, it is needed to make a updating of elastic parameters due to the interaction between elastic deformation and failure process. The set of parameters obtained for Tournemire shale are given in Table 1 and Table 2.

Table 1: Input parameters for three-dimensional sample generation

\begin{tabular}{lcc}
\hline Parameters used in $f(\phi, \theta)$ & Elastic parameters & Strength parameters \\
\hline Variables a, c & $0.1,0.1$ & $0.1,0.1$ \\
Variables $\mathrm{b}, f_{\max }$ & $1.23 * 10^{-2}, 9.0$ & $1.23 * 10^{-2}, 9.0$ \\
Variable $r_{k}$ & 1.0 & - \\
Variable $r_{s}$ & - & 0.15 \\
\hline
\end{tabular}

Table 2: Geometrical, physical and mechanical parameters for bond model and smooth joint model

\begin{tabular}{|c|c|c|c|}
\hline \multicolumn{4}{|l|}{ 3D Sample } \\
\hline Width of sample (mm) & $W$ & & 40.0 \\
\hline Height of sample (mm) & $H$ & & 80.0 \\
\hline Total grain number in sample & & & 20916 \\
\hline Average radius (mm) & $\bar{r}$ & & 1.0 \\
\hline Initial void ratio & & & 0.2 \\
\hline The reference radius (mm) & $R_{0}$ & & 2.0 \\
\hline Coefficient $r_{R}$ in function $f_{R}(\theta)$ & $r_{R}$ & & 0.5 \\
\hline Mechanical parameters & & Bond model & Smooth joint model \\
\hline Normal contact stiffness for test $(\mathrm{N} / \mathrm{m})$ & $k_{n}^{0}$ & $5.6 * 10^{8}$ & $8.0 * 10^{7}$ \\
\hline Shear contact stiffness for test $(\mathrm{N} / \mathrm{m})$ & $k_{s}^{0}$ & $2.8 * 10^{8}$ & $4.0 * 10^{7}$ \\
\hline Inter-particle coefficient of friction & $\tan \phi_{1}$ & 0.36 & 0.6 \\
\hline Inter-particle coefficient of friction & $\tan \phi_{2}$ & 1.9 & 1.3 \\
\hline Normal bond strength $(\mathrm{N})$ & $\varphi_{t 0}$ & $2.5 * 10^{4}$ & $0.42 * 10^{4}$ \\
\hline Shear bond strength $(\mathrm{N})$ & $\varphi_{s 0}$ & $8.0 * 10^{4}$ & $0.73 * 10^{4}$ \\
\hline The critical normal stress $(\mathrm{N})$ & $\varphi_{n c r}$ & $2.0 * 10^{5}$ & $1.5 * 10^{5}$ \\
\hline Inter-particle coefficient of friction & $\tan \phi_{r}$ & 0.05 & 0.05 \\
\hline
\end{tabular}

Note: These parameters for the smooth joint model are calculated with the average radius of weak layer zone $\bar{r}$.

\subsection{Experimental verification}

In order to verify the consistency of determined parameters, the macroscopic axial elastic modulus and peak stress of Tournemire shale are predicted by the proposed model. In Fig. 8(a), the variation of axial elastic modulus for two different confining stresses (5MPa and $50 \mathrm{MPa})$ are 
presented. One can see that the proposed model correctly reproduces the anisotropic property of macroscopic elastic modulus. In Fig. 8(b), the variation of peak stress with confining stress is presented for two loading orientations $\left(0^{\circ}\right.$ and $\left.45^{\circ}\right)$. It is found that the nonlinear pressure dependency of peak stress is well described by the unified bond and smooth joint model.

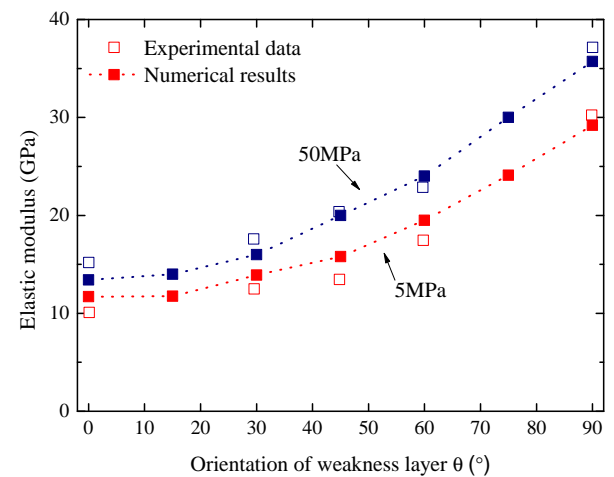

(a) Elastic modulus

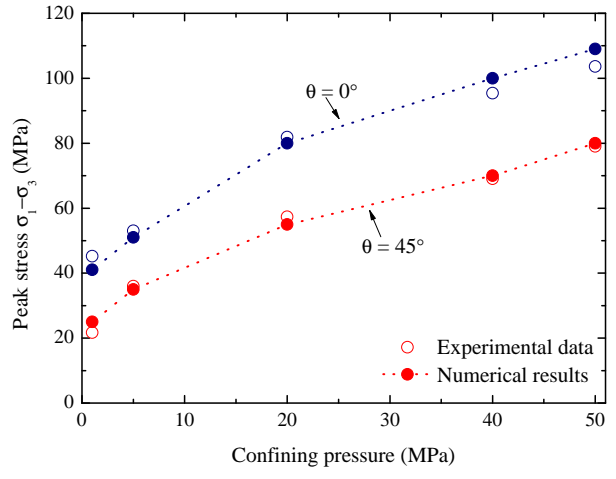

(b) Peak stress

Fig. 8: Comparison of axial elastic modulus and peak stress between numerical results and experiment data for Tournemire shale

In order to further check the performance of the numerical model, other numerical simulations are performed for five confining stresses (1MPa, 5MPa, 20MPa, 40MPa and 50MPa) and seven loading orientations (ranging from $0^{\circ}$ to $90^{\circ}$ ). The obtained numerical axial stressstrain curves are presented in Fig. 9. For all confining stresses, the strain-stress curves are clearly dependent on loading orientation. On the other hand, for a given loading orientation, the mechanical response is influenced by confining stress. In particular, for loading orientations close to $0^{\circ}$ or $90^{\circ}$, there is a clear transition from brittle to ductile behavior when the confining stress increases. This is a typical property of most rocks. For loading orientations around $45^{\circ}$, this transition seems less pronounced. For such orientations, the failure process of anisotropic rocks is strongly influenced by the frictional sliding along weak layers. With further experimental data, the smooth joint contact model can be improved to better capture this transition feature. For a quantitative comparison, the numerical values of peak differential stress are compared with experimental data in Fig. 9(e). A very good agreement is obtained. For all loading orientations, the peak stress as well as the residual stress increase when the confining stress is higher. There are two maximum values of peak stress obtained either at $0^{\circ}$ or $90^{\circ}$. The minimum value of peak stress occurs between $30^{\circ}$ and $60^{\circ}$ for all confining stresses. For convenience, two strength anisotropy coefficients are usually defined as follows:

$$
K_{1}=\frac{\left(\sigma_{1}-\sigma_{3}\right)_{\|}}{\left(\sigma_{1}-\sigma_{3}\right)_{\perp}}
$$




$$
K_{2}=\frac{\left(\sigma_{1}-\sigma_{3}\right)_{\max }}{\left(\sigma_{1}-\sigma_{3}\right)_{\min }}
$$

$K_{1}$ defines the ratio between the peak stresses in the principal directions, respectively parallel and perpendicular to the weak layers, and $K_{2}$ gives the ratio between the maximum and minimum peak stress. The values of these two coefficients for Tournemire shale under different confining stresses are shown in Fig. 10. The value of $K_{1}$ is nearly constant and equal to 1.0, indicating that the strength difference between the two principal directions is very small. The value of $K_{2}$ decreases from 1.96 to 1.47 with the increase of confining stress from $1 \mathrm{MPa}$ to $50 \mathrm{MPa}$. This implies that the strength anisotropy is attenuated when the confining stress becomes high. These numerical results are also consistent with the experimental data obtained from other anisotropic rocks [11]. 


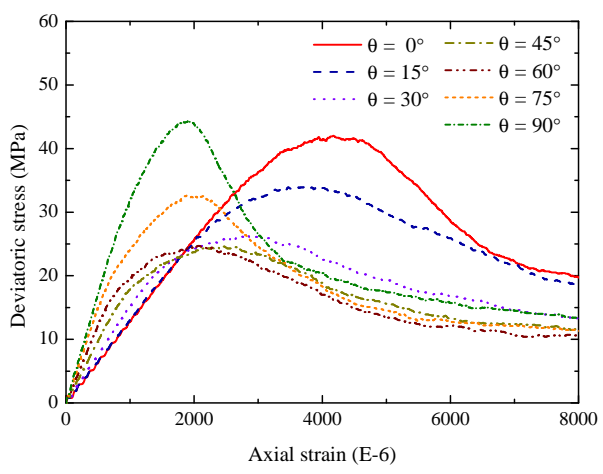

(a) $1 \mathrm{MPa}$

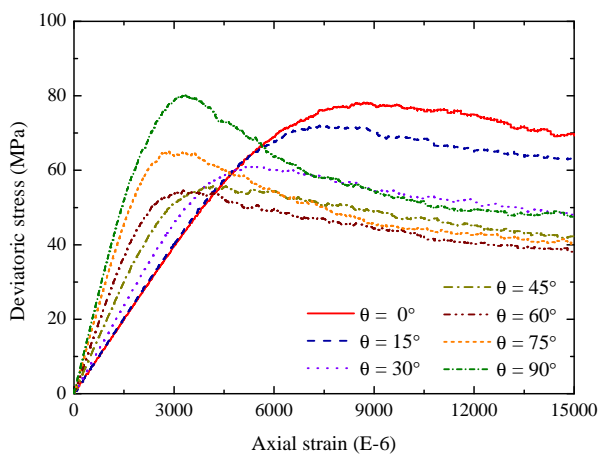

(c) $20 \mathrm{MPa}$

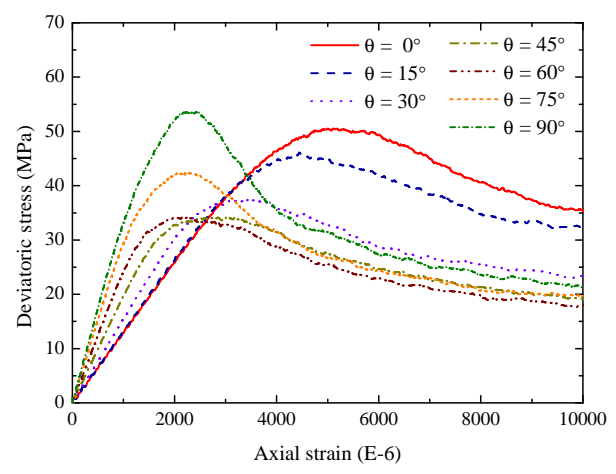

(b) $5 \mathrm{MPa}$

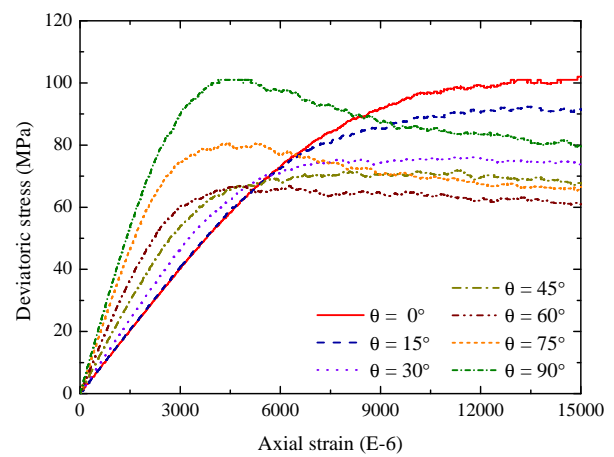

(d) $40 \mathrm{MPa}$

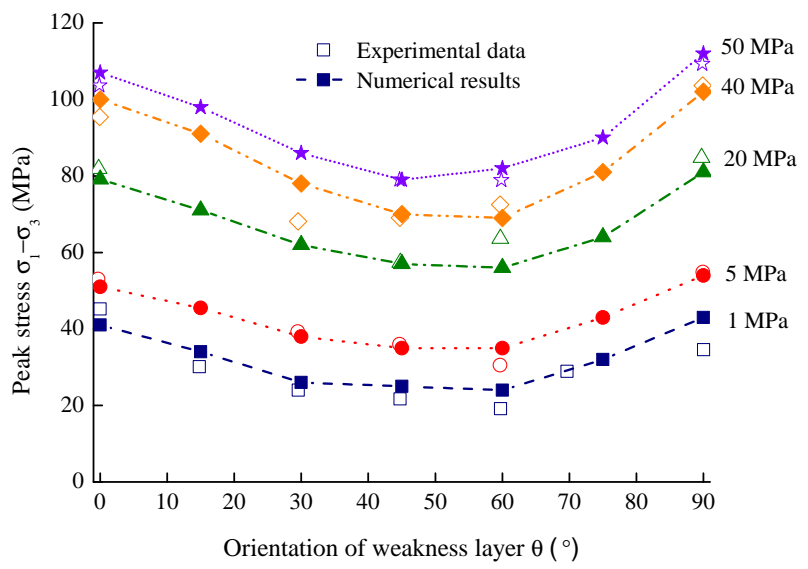

(e) Variation of peak differential stress

Fig. 9: Stress-strain curves and peak differential stress for Tournemire shale samples with different loading orientations and under various confining stresses 


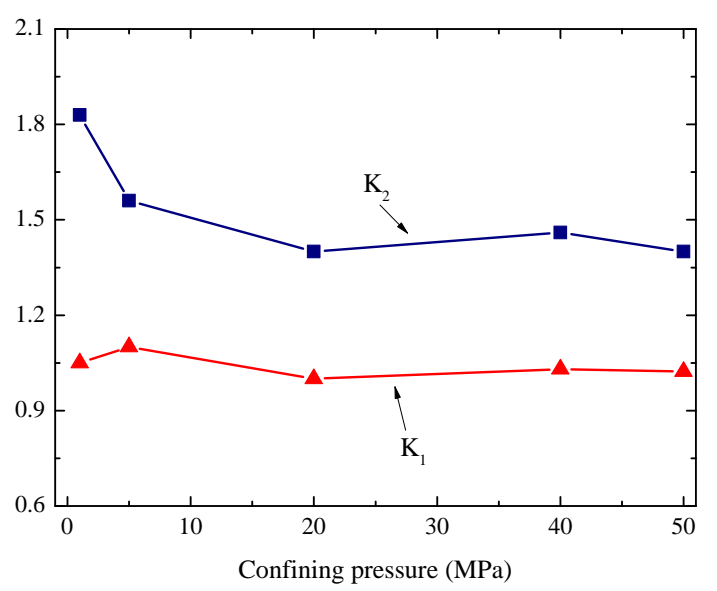

Fig. 10: Variation of anisotropy coefficients of mechanical strength versus confining stress

\section{Further analysis of deformation and failure process}

After the description of macroscopic stress-strain responses of anisotropic rocks in the previous section, the spatial distribution of deformation and broken contacts in three dimensional specimens are now discussed.

\subsection{Spatial distribution of micro-cracks}

We adopt here the terminology commonly used for rock-like materials. When a cohesive contact between two particles is broken according to the failure criteria defined above, the contact is seen as a micro-crack.

In Fig. 11, we show the stress-strain curves and the evolution of broken contact numbers in triaxial compression tests with three loading orientations $\left(0^{\circ}, 45^{\circ}, 90^{\circ}\right)$ and two confining stresses $(1 \mathrm{MPa}$ and $40 \mathrm{MPa})$. In order to better capture the failure process of samples in the different tests, four characteristic stages are identified on the stress-strain curve of each test. The stage 1 corresponds to the initiation of micro-cracks, the stage 2 is defined by the peak differential stress, the stage 3 is related to the coalescence of micro-cracks and the stage 4 represents the onset of macroscopic cracks and the beginning of residual strength phase. In this study, we put the emphasis on the macroscopic cracking stage. Therefore, unless specified, all the results presented in the subsequent figures are collected at the stage 4, namely at the points A4, B4, C4, D4, E4 and F4 shown in Fig. 11.

It is found that the local cracking process is both influenced by confining stress and loading orientation. In a general way and for two confining stresses considered here, in the matrix zone, the number of tensile cracks is systematically higher than that of shear cracks. For the low confining stress of $1 \mathrm{MPa}$, the number of shear cracks is very small. It increases when 

and two confining stresses. It is interesting to observe that the spatial orientations of cracked bonds are strongly correlated with the loading orientation with respect to the weak layers. 


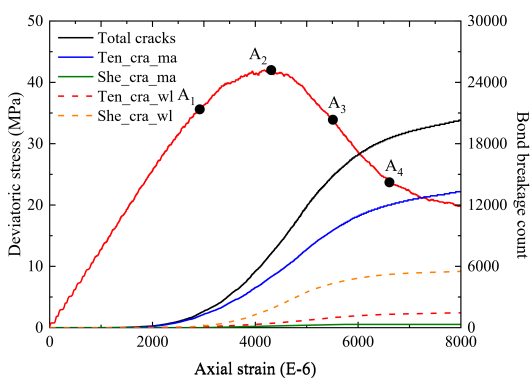

(a) $\theta=0^{\circ}$

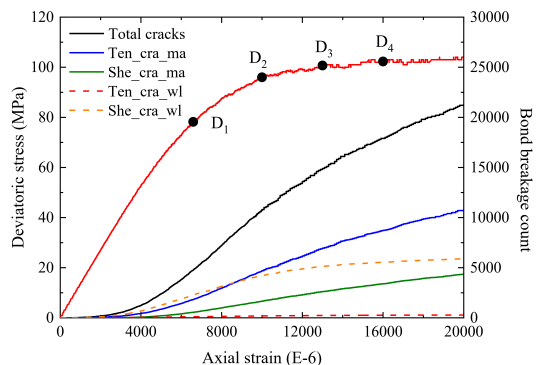

(d) $\theta=0^{\circ}$

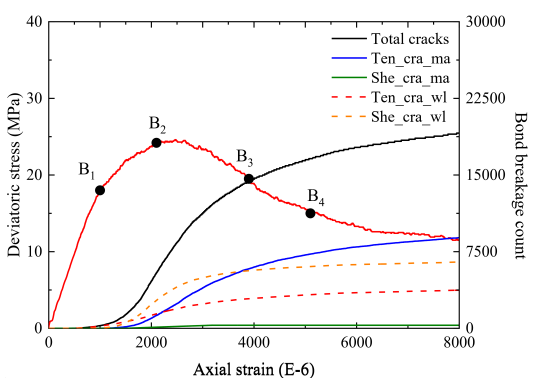

(b) $\theta=45^{\circ}$

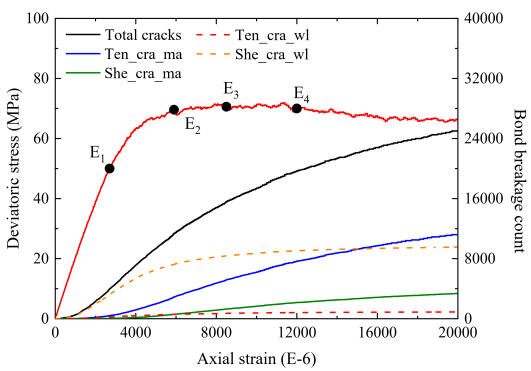

(e) $\theta=45^{\circ}$

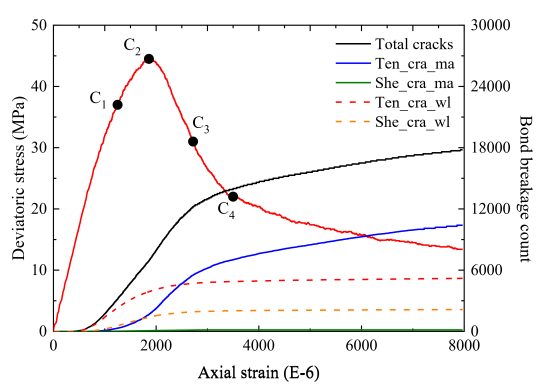

(c) $\theta=90^{\circ}$

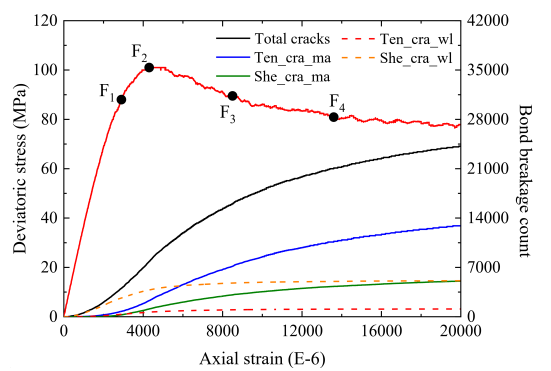

(f) $\theta=90^{\circ}$

Fig. 11: Number evolution of different kinds of micro-cracks for three selected loading orientations and two confining stresses of $1 \mathrm{MPa}$ and $40 \mathrm{MPa}$ (black for total crack number, red and yellow respectively for tensile and shear cracks in weak layers, blue and green respectively for tensile and shear cracks in matrix phase)

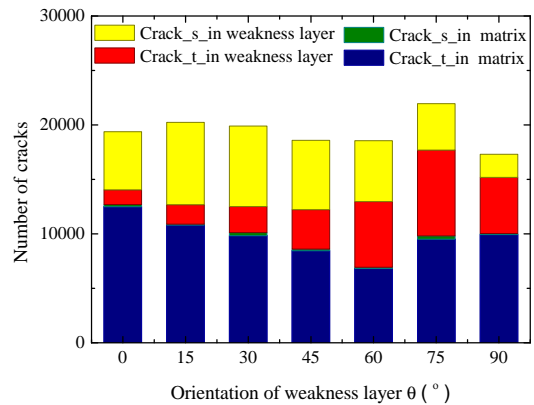

(a) $1 M P a$

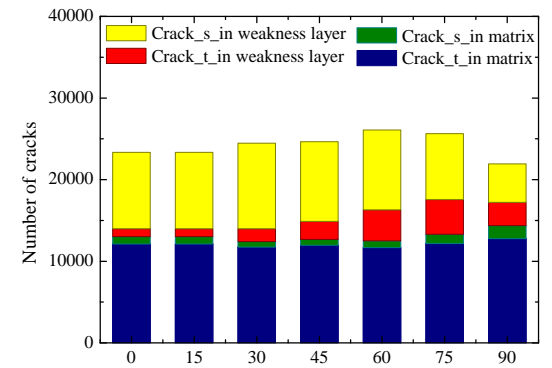

Orientation of weakness layer $\theta\left({ }^{\circ}\right)$

(b) $20 M P a$

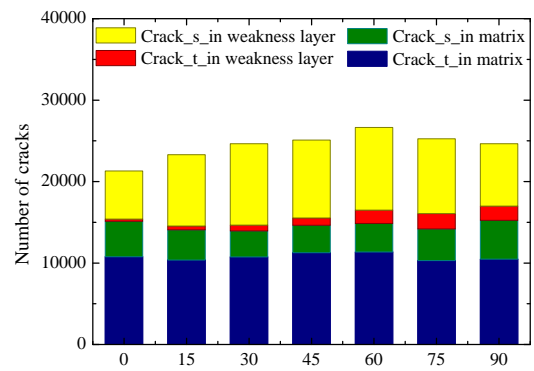

Orientation of weakness layer $\theta\left({ }^{\circ}\right)$

(c) $40 \mathrm{MPa}$

Fig. 12: Repartition of micro-cracks in matrix phase and weak layers for different loading orientations and confining stresses 


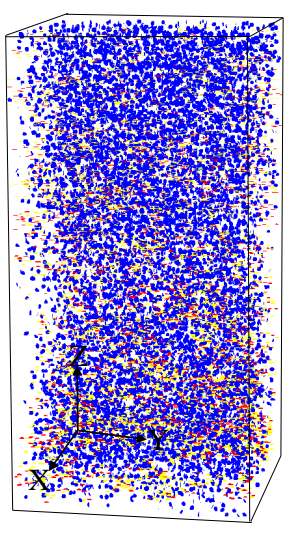

$\theta=0^{\circ}$

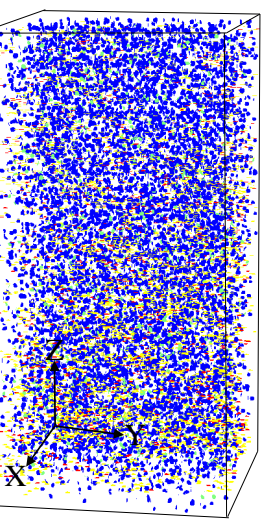

$\theta=0^{\circ}$

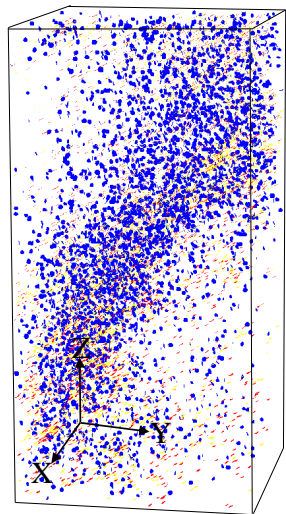

$\theta=30^{\circ}$

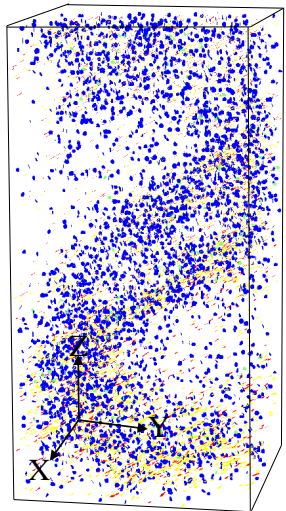

$\theta=30^{\circ}$

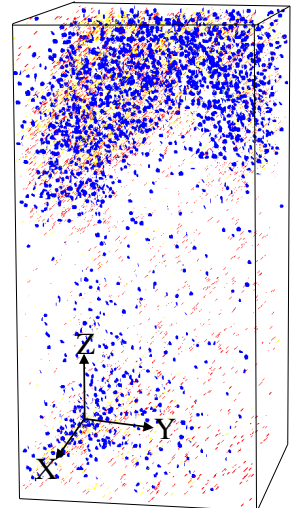

$\theta=45^{\circ}$

(a) $1 M P a$

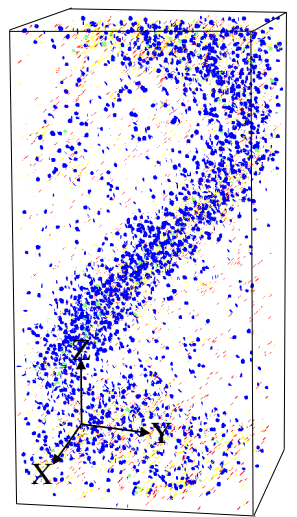

$\theta=45^{\circ}$

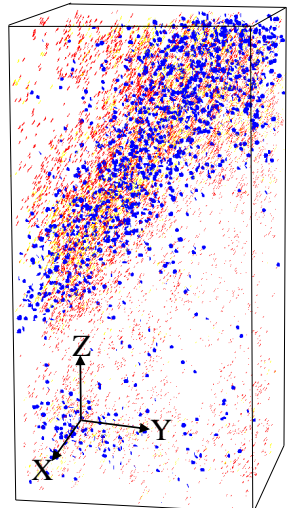

$\theta=60^{\circ}$

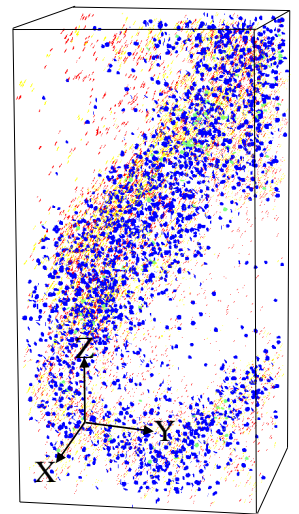

$\theta=60^{\circ}$

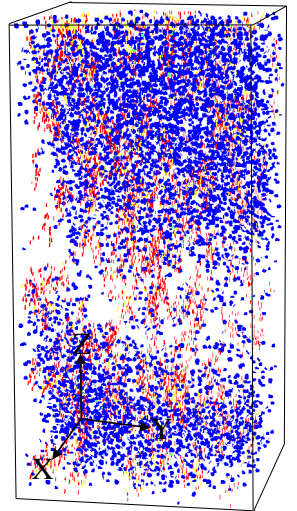

$\theta=90^{\circ}$

(b) $20 M P a$

Fig. 13: Spatial distribution of cracked contacts for different loading orientations under two different confining stresses (red and yellow for tensile and shear cracks in weak layers; blue and green for tensile and shear cracks in matrix phase) 

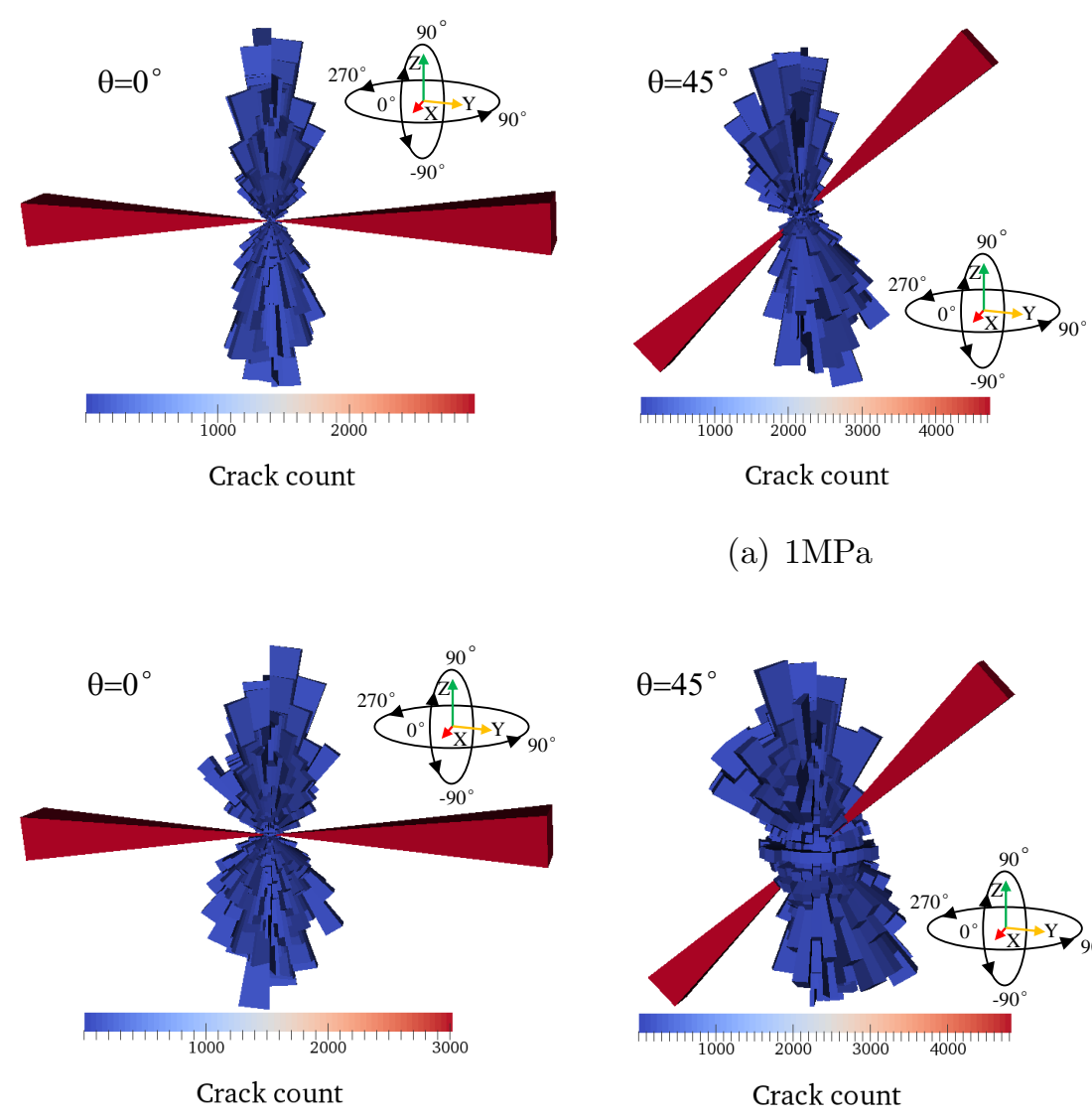

(a) $1 \mathrm{MPa}$
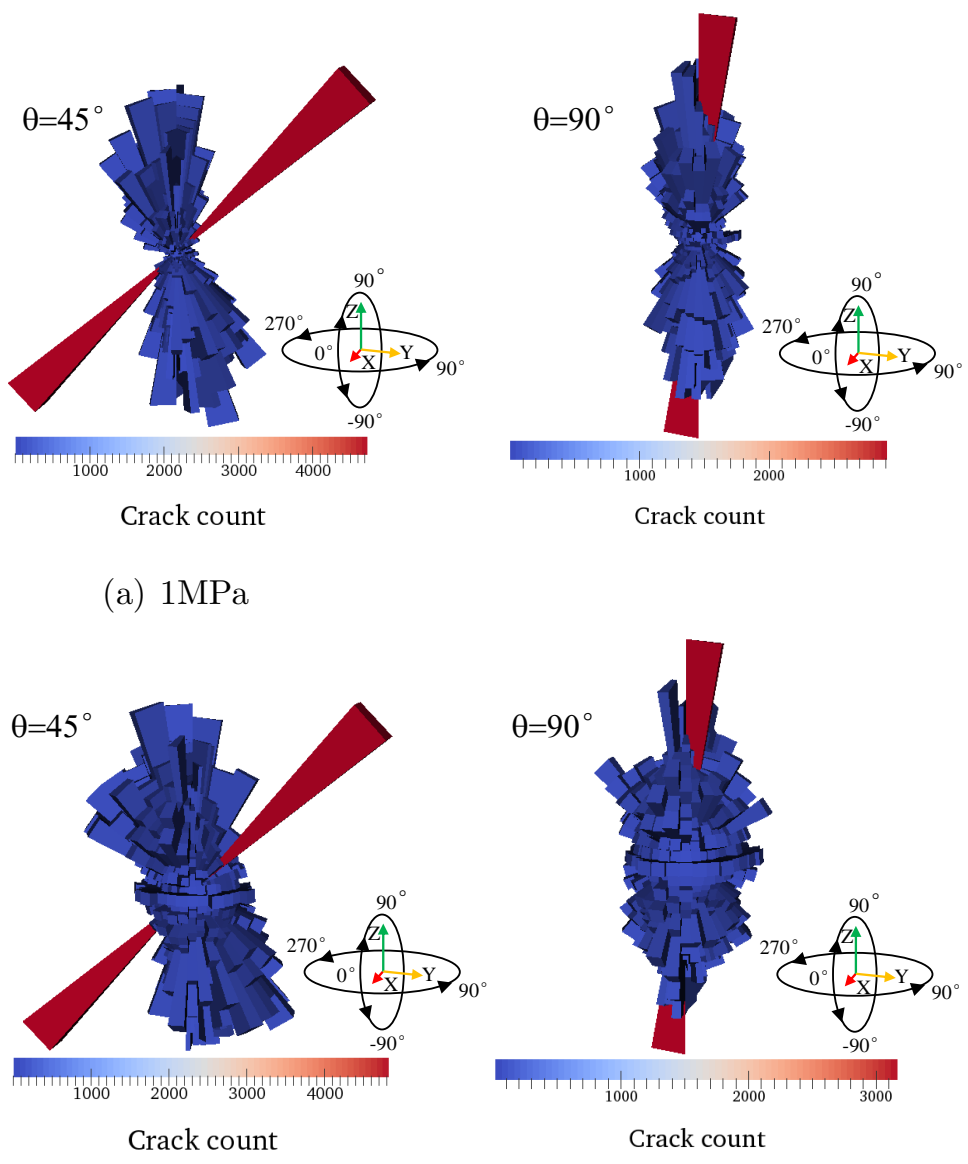

(b) $40 \mathrm{MPa}$

Fig. 14: Spatial distribution of cracked contacts orientations for different loading orientations under low and high confining stresses (blue for matrix phase; red for weak layers)

\subsection{Spatial distribution of displacement}

On the other hand, the spatial distribution of particles displacements inside three dimensional samples is evaluated and shown in Fig. 15(a). Notice that the bottom surface of samples is blocked in the vertical direction. According to these results, it seems that the displacement pattern is more significantly influenced by loading orientation than by the confining stress. For instance, the displacement distributions are very similar for two principal orientations, $0^{\circ}$ and $90^{\circ}$, for which the axial stress is respectively perpendicular and parallel to the weak layers. One obtains a quasi vertical gradient of displacement for these two cases. For other loading orientations in which the weak layers are inclined to the axial stress. The displacement distribution is clearly affected by the presence of weak layers and one obtains an inclined displacement gradient. We can distinguish two blocks. The upper one is sliding with respect to the lower one along an inclined direction. However, the spatial distribution of displacement does not provide an obvious description of macroscopic failure process of samples. For this reason, we introduce 
the following displacement localization coefficient, which is defined by:

$$
F_{c o e}=\frac{\left|D_{i+1}-D_{i}\right|}{\left|\left(D_{i+1}-D_{i}\right)_{\max }\right|}
$$

The quantity $\left(D_{i+1}-D_{i}\right)$ defines the displacement difference between two adjacent particles, little affected by the confining stress. 


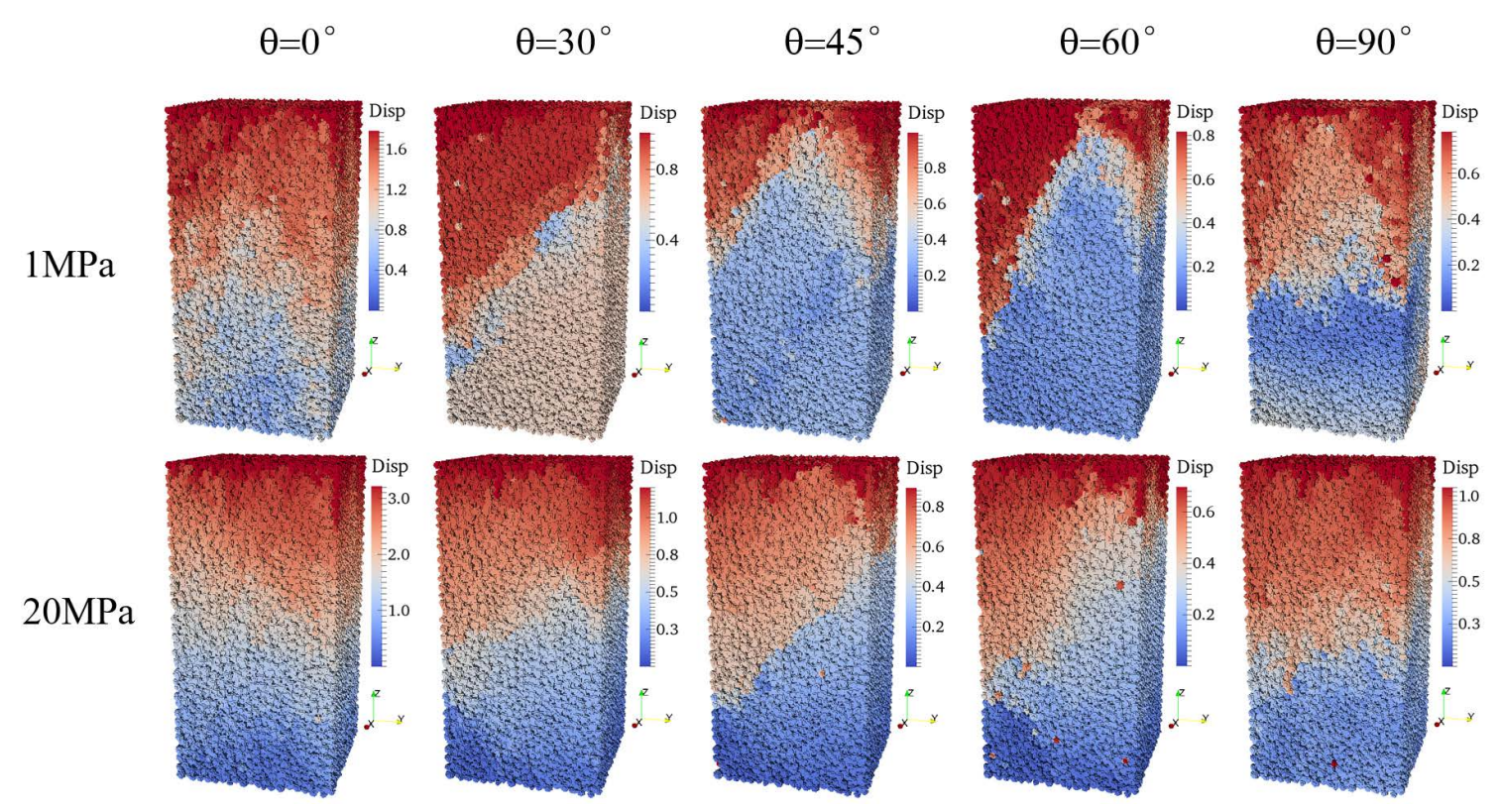

(a) Displacement

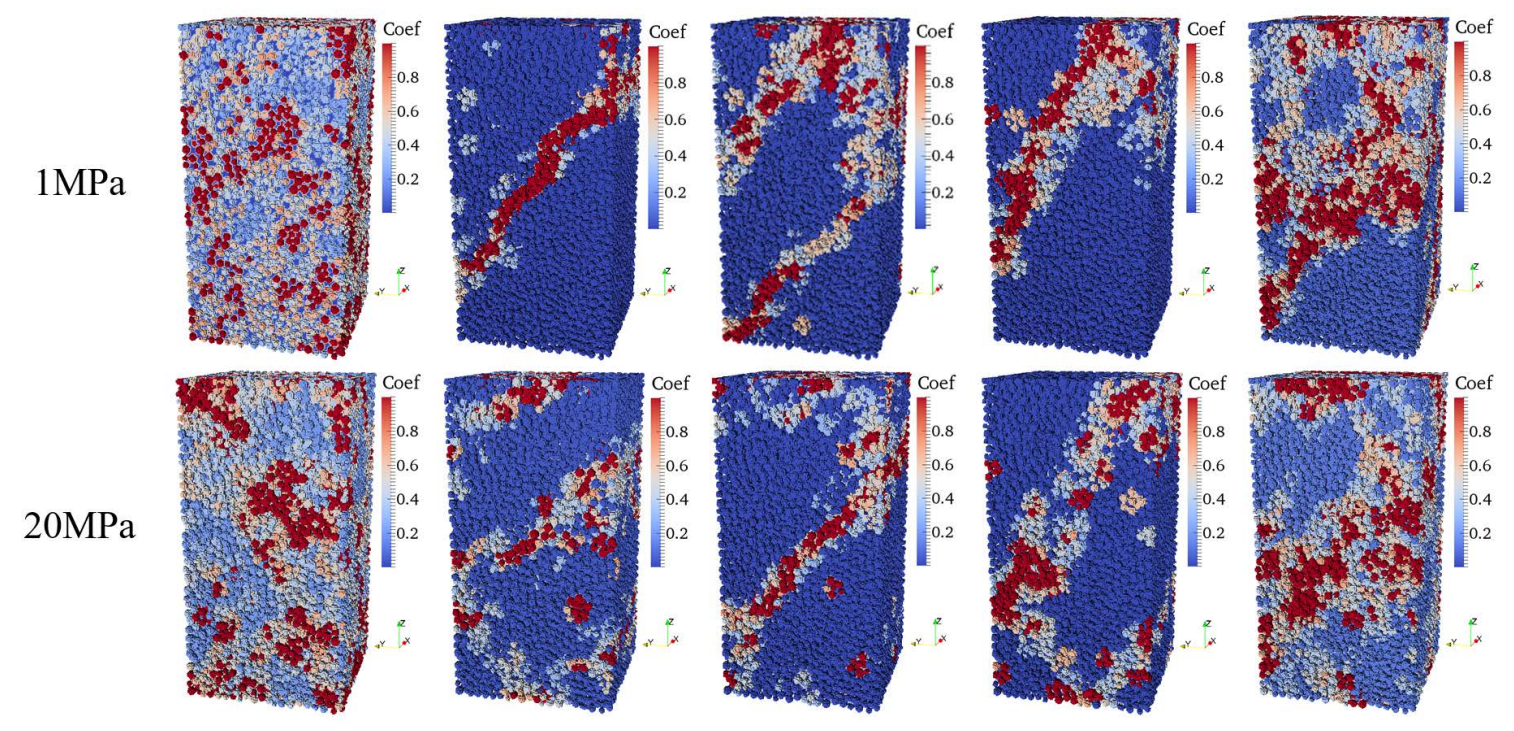

(b) Displacement localization coefficient

Fig. 15: Spatial distribution of displacement and displacement localization coefficient in samples for different loading orientations and confining stresses

\section{Concluding remarks}

In this paper, we have developed a new particle-based model for modeling deformation and strength properties of anisotropic rocks. A numerical procedure has been proposed to generate oriented weak layers in a three dimensional rock sample. Each anisotropic sample is composed of bond contacts in the matrix phase and smooth joint contacts in the weak layers. We have further proposed a unified nonlinear model for the description of tensile and shear failure for both the bond and smooth joint contacts. The comparison between numerical predictions and 
[4] Yong Ming Tien, Ming Chuan Kuo, and Charng Hsein Juang. An experimental investigation of the failure mechanism of simulated transversely isotropic rocks. International Journal of Rock Mechanics and Mining Sciences, 43(8):1163-1181, 2006. of confining stress on the elastic and strength properties of anisotropic rocks. The cracking process at the microscopic scale has been studied. In a general way, the failure of matrix phase is dominated by the tensile cracking while the shear cracking becomes more and more important when the confining stress increases. In the weak layers, the shear cracking is the dominant process, in particular under a high confining stress. Under a low confining stress, the tensile cracking plays an important role. For a given confining stress, the tensile cracking in the weak layers is more important when the loading direction is parallel to the weak layers. About the macroscopic failure mode of samples, it is found that when the loading direction is either parallel or perpendicular to the weak layers, one obtains a diffuse failure mode. For other situations, significant inclined localization bands can be formed. In particular, when the inclination angle of weak layers is between $45^{\circ}$ and $60^{\circ}$, the localized bands are nearly parallel to the weak layers. In our future studies, we shall investigate time-dependent deformation and failure as well as hydromechanical interactions.

\section{Acknowledgement}

This study was partially supported by the National Key RD Program of China (Grant No. 2017YFC1501100).

\section{References}

[1] H Niandou, JF Shao, JP Henry, and D Fourmaintraux. Laboratory investigation of the mechanical behaviour of tournemire shale. International Journal of Rock Mechanics and Mining Sciences, 34(1):3-16, 1997.

[2] Jung-Woo Cho, Hanna Kim, Seokwon Jeon, and Ki-Bok Min. Deformation and strength anisotropy of asan gneiss, boryeong shale, and yeoncheon schist. International Journal of Rock Mechanics and Mining Sciences, 50:158-169, 2012.

[3] Erling Fjær and Olav-Magnar Nes. The impact of heterogeneity on the anisotropic strength of an outcrop shale. Rock mechanics and rock engineering, 47(5):1603-1611, 2014. 
[5] Abbass Tavallali and André Vervoort. Effect of layer orientation on the failure of layered sandstone under brazilian test conditions. International journal of rock mechanics and mining sciences, 47(2):313-322, 2010.

[6] Bjorn Debecker and André Vervoort. Two-dimensional discrete element simulations of the fracture behaviour of slate. International Journal of Rock Mechanics and Mining Sciences, 61:161-170, 2013.

[7] JC Jaeger. Shear failure of anistropic rocks. Geological Magazine, 97(1):65-72, 1960.

[8] Evert Hoek and Edwin T Brown. Underground excavations in rock. CRC Press, 1980.

[9] R Nova. The failure of transversely isotropic rocks in triaxial compression. In International Journal of Rock Mechanics and Mining Sciences 8 Geomechanics Abstracts, volume 17, pages 325-332. Elsevier, 1980.

[10] Bernard Amadei and William Z Savage. Anisotropic nature of jointed rock mass strength. Journal of Engineering Mechanics, 115(3):525-542, 1989.

[11] G Duveau, JF Shao, and JP Henry. Assessment of some failure criteria for strongly anisotropic geomaterials. Mechanics of Cohesive-frictional Materials: An International Journal on Experiments, Modelling and Computation of Materials and Structures, 3(1):1$26,1998$.

[12] JP Boehler and J Raclin. Failure criteria for glass-fiber reinforced composites under confining pressure. J. Struct. Mech., 13:371-393, 1976.

[13] JP Boehler and A Sawczuk. On yielding of orientated solids. Acta Mechanica, 27:185-206, 1977.

[14] O Cazacu, ND Cristescu, JF Shao, and JP Henry. A new anisotropic failure criterion for transversely isotropic solids. Mechanics of cohesive frictional materials, 3:98-103, 1998.

[15] Youn-Kyou Lee and S Pietruszczak. Application of critical plane approach to the prediction of strength anisotropy in transversely isotropic rock masses. International Journal of Rock Mechanics and Mining Sciences, 45(4):513-523, 2008.

[16] S Pietruszczak, D Lydzba, and Jian-Fu Shao. Modelling of inherent anisotropy in sedimentary rocks. International Journal of Solids and Structures, 39(3):637-648, 2002. 
[17] Peter A Cundall and Otto DL Strack. A discrete numerical model for granular assemblies. geotechnique, 29(1):47-65, 1979.

[18] DO Potyondy and PA Cundall. A bonded-particle model for rock. International journal of rock mechanics and mining sciences, 41(8):1329-1364, 2004.

[19] MJ Jiang, HB Yan, HH Zhu, and S Utili. Modeling shear behavior and strain localization in cemented sands by two-dimensional distinct element method analyses. Computers and Geotechnics, 38(1):14-29, 2011.

[20] Sheng-Qi Yang, Yan-Hua Huang, Hong-Wen Jing, and Xiang-Ru Liu. Discrete element modeling on fracture coalescence behavior of red sandstone containing two unparallel fissures under uniaxial compression. Engineering geology, 178:28-48, 2014.

[21] Mohammad Hadi Mehranpour and Pinnaduwa HSW Kulatilake. Comparison of six major intact rock failure criteria using a particle flow approach under true-triaxial stress condition. Geomechanics and Geophysics for Geo-Energy and Geo-Resources, 2(4):203-229, 2016.

[22] Peng-fei He, Pinnaduwa HSW Kulatilake, Xu-xu Yang, Dong-qiao Liu, and Man-chao He. Detailed comparison of nine intact rock failure criteria using polyaxial intact coal strength data obtained through pfc3d simulations. Acta Geotechnica, pages 1-27, 2017.

[23] Weijiang Chu, Chunsheng Zhang, Jing Hou, et al. A particle-based model for studying anisotropic strength and deformation of schist. In ISRM SINOROCK 2013, pages 60-69. International Society for Rock Mechanics and Rock Engineering, 2013.

[24] CY Kwok, K Duan, LG Tham, et al. Numerical simulation of strength and deformation behavior of inherently anisotropic rocks. In 48th US Rock Mechanics/Geomechanics Symposium. American Rock Mechanics Association, 2014.

[25] K Duan and CY Kwok. Discrete element modeling of anisotropic rock under brazilian test conditions. International Journal of Rock Mechanics and Mining Sciences, 2015.

[26] Bona Park and Ki-Bok Min. Bonded-particle discrete element modeling of mechanical behavior of transversely isotropic rock. International Journal of Rock Mechanics and Mining Sciences, 76:243-255, 2015. 
[27] K Duan, CY Kwok, and M Pierce. Discrete element method modeling of inherently anisotropic rocks under uniaxial compression loading. International Journal for Numerical and Analytical Methods in Geomechanics, 40(8):1150-1183, 2016.

[28] Mohammad Hadi Mehranpour and Pinnaduwa HSW Kulatilake. Improvements for the smooth joint contact model of the particle flow code and its applications. Computers and Geotechnics, 87:163-177, 2017.

[29] Toivo Wanne. Rock strength and deformation dependence on schistosity. Simulation of rock with PFC3D. Report, 5, 2002.

[30] Bona Park, Ki-Bok Min, Nicholas Thompson, and Per Horsrud. Three-dimensional bonded-particle discrete element modeling of mechanical behavior of transversely isotropic rock. International Journal of Rock Mechanics and Mining Sciences, 110:120 - 132, 2018.

[31] Diego Mas Ivars, Matthew E Pierce, Caroline Darcel, Juan Reyes-Montes, David O Potyondy, R Paul Young, and Peter A Cundall. The synthetic rock mass approach for jointed rock mass modelling. International Journal of Rock Mechanics and Mining Sciences, 48(2):219-244, 2011.

[32] Chia-Chi Chiu, Tai-Tien Wang, Meng-Chia Weng, and Tsan-Hwei Huang. Modeling the anisotropic behavior of jointed rock mass using a modified smooth-joint model. International Journal of Rock Mechanics and Mining Sciences, 62:14-22, 2013.

[33] Tao Wang, Dapeng Xu, Derek Elsworth, and Weibo Zhou. Distinct element modeling of strength variation in jointed rock masses under uniaxial compression. Geomechanics and Geophysics for Geo-Energy and Geo-Resources, 2(1):11-24, 2016.

[34] K Duan and CY Kwok. Evolution of stress-induced borehole breakout in inherently anisotropic rock: Insights from discrete element modeling. Journal of Geophysical Research: Solid Earth, 121(4):2361-2381, 2016.

[35] Jian Zhou, Luqing Zhang, Zhejun Pan, and Zhenhua Han. Numerical studies of interactions between hydraulic and natural fractures by smooth joint model. Journal of Natural Gas Science and Engineering, 46:592-602, 2017.

[36] Yulong Zhang, Jianfu Shao, Zaobao Liu, Chong Shi, and Géry De Saxcé. Effects of confining pressure and loading path on deformation and strength of cohesive granular materials: a three-dimensional dem analysis. Acta Geotechnica, pages 1-18, 2018. 
[37] CG Itasca. Pfc 3d-user manual. Itasca Consulting Group, Minneapolis, 1999.

[38] PFC3D Manuals. Pfc 3d-user manual, verison 4.0 itasca consulting group inc. Minneapolis, MI, 2008.

[39] Yan H Jiang, MJ and Utili S Zhu, H. Modeling shear behavior and strain localization in cemented sands by two-dimensional distinct element method analyses. Computers and Geotechnics, 38:14-29, 2011.

[40] X Ding and L Zhang. A new contact model to improve the simulated ratio of unconfined compressive strength to tensile strength in bonded particle models. International Journal of Rock Mechanics and Mining Sciences, 69:111-119, 2014.

[41] C Yao, QH Jiang, JF Shao, and CB Zhou. A discrete approach for modeling damage and failure in anisotropic cohesive brittle materials. Engineering Fracture Mechanics, 155:102$118,2016$.

[42] Chi Yao, QH Jiang, and Jian-Fu Shao. Numerical simulation of damage and failure in brittle rocks using a modified rigid block spring method. Computers and Geotechnics, 64:48-60, 2015.

450

[43] MH Mehranpour and PH Kulatilake. Comparison of six major intact rock failure criteria using a particle flow approach under true-triaxial stress condition. Geomechanics and Geophysics for Geo-Energy and Geo-Resources, 2:203-229, 2016. 